\title{
Short-term effects of repetitive transcranial magnetic stimulation on sleep bruxism - a pilot study
}

\author{
Wei-Na Zhou ${ }^{1}$, Hai-Yang Fu ${ }^{2}$, Yi-Fei Du ${ }^{3}$, Jian-Hua Sun ${ }^{2}$, Jing-Lu Zhang ${ }^{1}$, Chen Wang ${ }^{1}$, Peter Svensson ${ }^{4}$ and \\ Ke-Lun Wang ${ }^{1,5}$
}

The purpose of this study was to investigate the effects of repetitive transcranial magnetic stimulation (rTMS) on patients with sleep bruxism (SB). Twelve patients with SB were included in an open, single-intervention pilot study. rTMS at $1 \mathrm{~Hz}$ and an intensity of $80 \%$ of the active motor threshold was applied to the 'hot spot' of the masseter muscle representation at the primary motor cortex bilaterally for $\mathbf{2 0} \mathrm{min}$ per side each day for 5 consecutive days. The jaw-closing muscle electromyographic (EMG) activity during sleep was recorded with a portable EMG recorder at baseline, during rTMS treatment and at follow-up for 5 days. In addition, patients scored their jaw-closing muscle soreness on a 0-10 numerical rating scale (NRS). Data were analysed with analysis of variance. The intensity of the EMG activity was suppressed during and after rTMS compared to the baseline $(P=0.04 ; P=0.02$, respectively).

The NRS score of soreness decreased significantly during and after rTMS compared with baseline $(P<0.01)$. These findings indicated a significant inhibition of jaw-closing muscle activity during sleep along with a decrease of muscle soreness. This pilot study raises the possibility of therapeutic benefits from rTMS in patients with bruxism and calls for further and more controlled studies.

International Journal of Oral Science (2016) 8, 61-65; doi:10.1038/ijos.2015.35; published online 13 November 2015

Keywords: behavioural science; brain function; electromyography; orofacial pain; repetitive transcranial magnetic stimulation; sleep bruxism

\section{INTRODUCTION}

Sleep bruxism $(\mathrm{SB})$ is defined as a repetitive jaw-closing muscle activity characterised by clenching or grinding the teeth and/or by bracing or thrusting the mandible during sleep. ${ }^{1} \mathrm{SB}$ is thought to be potentially harmful to the stomatognathic system because it causes, for example, excessive tooth wear, masseter hypertrophy, temporomandibular disorders (TMD) and headaches. ${ }^{2-4}$ The aetiology of SB is complex but most likely includes arousal responses and autonomic dysregulation, genetic, psycho-emotional and pharmacological risk factors. ${ }^{5}$

In recent years, the central nervous system factors have been emphasised over the peripheral factors, such as occlusal discrepancies and deviations, in bony structures of the orofacial region. ${ }^{5-6} \mathrm{SB}$ may be considered an extreme manifestation of the masticatory muscle activity that occurs during sleep in most individuals. ${ }^{6-7}$ Interestingly, a neuroimaging study suggested an association between bruxism and a dysfunction in the central regulation of jaw movements. The onset of rhythmic masticatory muscle activity (RMMA) and SB episodes was suggested to be influenced by brief and transient arousal responses in the brainstem activity according to a polysomnographic (PSG) study. ${ }^{8}$ A magnetoencephalography study indicated that SB patients had significantly larger event-related desynchronisation in the sensory-motor area than did non-bruxism subjects. ${ }^{9}$ A sequential change from autonomic (cardiac) and cortical activities preceded SB-related jaw motor activity, and repetitive stimulation to the primary face motor cortex was shown to induce rhythmic jaw movements in non-human primate studies. ${ }^{10}$ Accordingly, SB patients might exhibit a dysfunction of motor-related subcortical and cortical networks.

Traditional treatment strategies of SB include occlusal, behavioural, physical and pharmacological approaches. ${ }^{11}$ However, no permanent suppression of jaw-closing muscle activity has yet been demonstrated, and reversible and non-invasive approaches are therefore preferred. . $^{2,11-12}$

Transcranial magnetic stimulation (TMS) has been widely used in humans to assess the motor cortex connection to a number of target muscles, ${ }^{13-14}$ and in recent years, repetitive applications of TMS (rTMS) have been used to study the inhibitory or excitatory influence on a variety of cortical functions. Low-frequency $(1 \mathrm{~Hz})$ rTMS has been found to inhibit neuronal firing in a localised cortical area, whereas high-frequency rTMS inversely led to neuronal depolarisation under the stimulating coil. rTMS has now been clinically applied for management of depression, pain and tinnitus, ${ }^{14-15}$ but to date, no study has examined its effect on SB. Based on the reviewed literature, it

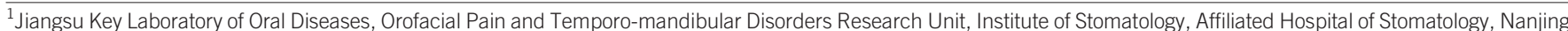

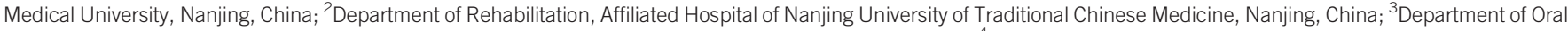

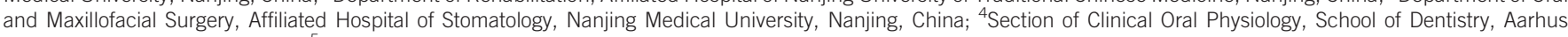
University, Aarhus, Denmark and ${ }^{5}$ Center for Sensory-Motor Interaction (SMI), Aalborg University, Aalborg, Denmark

Correspondence: Dr JL Zhang, Jiangsu Key Laboratory of Oral Diseases, Orofacial Pain and temporo-mandibular Disorders Research Unit, Institute of Stomatology, Affiliated Hospital of Stomatology, Nanjing Medical University, 136 Hanzhong Road, Nanjing 210029, China

E-mail: zhjllym@njmu.edu.cn

Accepted 31 August 2015 
seems plausible that rTMS of the primary sensory-motor cortex representation of the jaw-closing muscle could have a beneficial effect on SB.

In the present pilot study, we attempted to use rTMS to suppress the corticobulbar pathways and inhibit the jaw-closing muscle activity during sleep. Potential clinical effects were examined by assessing self-reported masseter muscle soreness. An open, single-intervention trial design was applied to provide preliminary evidence of treatment effects before the initiation of a randomised clinical trial. Thus, the purpose of this study was to investigate the possible effects of rTMS on SB.

\section{MATERIALS AND METHODS}

\section{Ethics}

The study was approved by the Human Research Ethics Committee at Nanjing Medical University. All patients completed a modified version of the rTMS Adult Safety Screen Questionnaire to ensure that there was no contraindication for rTMS.

\section{Patients}

All participants were selected from patients referred to the TMD Clinic, Stomatology Hospital of Jiangsu province, China. Sixty patients with probable SB were initially evaluated, and 18 patients were eligible and agreed to participate in the experiment. After one week, six patients withdrew from the study for personal reasons. A total of 12 individuals ( 7 men and 5 women, age: $(27 \pm 5.4)$ years $)$ completed the study.

The same dentist administered the questionnaire and performed the clinical assessment on all patients in accordance with a diagnosis of 'probable' SB. ${ }^{1}$ The patients were aware of their tooth-grinding activity during sleep or had been told of it by a bed partner. Furthermore, there were positive findings for one or more of the followings signs or symptoms: (i) tooth wear and/or shiny spots on dental restorations; (ii) frequent reports of stiffness, fatigue or discomfort in the jawclosing muscles upon awakening; and (iii) masseter muscle hypertrophy during voluntary contractions. ${ }^{16}$

The exclusion criteria were a history of neurologic or psychiatric disorders; the use of prescription medicine or drugs with possible sleep effects or alterations of motor behaviour; an electrode gel allergy; currently being under medical or dental treatment; having a pacemaker or implanted defibrillator; the loss of more than two posterior teeth except third molars; or having removable partial or full dentures.

\section{Study design}

A pilot study with an open, single-intervention design with three phases of investigation was chosen. In phase 1, all participants used a portable, single-channel electromyographic (EMG) recorder in their own home to record baseline jaw-closing muscle EMG activity during sleep for five nights. In phase 2, all participants received rTMS treatment during the day for at least five consecutive days, and the jawclosing muscle EMG activity was recorded during sleep on the nights following treatment. In phase 3 , all participants were followed-up with immediately after the last day of rTMS treatment and again used the portable EMG recorder for at least five nights.

At the end of each phase, all participants were asked to evaluate the level of soreness in the jaw-closing muscles by choosing a score on a numerical rating scale (NRS), where 0 represented no soreness and 10 represented the most imaginable soreness.

\section{EMG recordings}

A single-channel portable EMG device (Grindcare3; Medotech A/S, Herlev, Denmark) was used to record EMG activity during sleep. All recordings were performed in the subjects' homes. The device has a single electrode assembly with three electrode contacts. The electrodes are designed to be placed over the anterior temporalis muscle, which is one of the jaw-closing muscles and may provide the same type of EMG information as obtained from the masseter muscle during sleep. The EMG activity was recorded through the amplifiers ( $\times 800$ times) and filters $(250-610 \mathrm{~Hz})$ in the device and further analysed for events of EMG activity using a Signal Recognition Algorithm described by Jadidi et al. ${ }^{17}$ To determine the individual parameters, the following set-up procedure was performed every night: patients were asked to relax their jaw-closing muscle for $10 \mathrm{~s}$. Then, they clenched their teeth at maximal voluntary clenching (MVC) for $10 \mathrm{~s}$, and $60 \%$ of MVC was pre-set for the threshold of starting the EMG recording. These procedures were carefully demonstrated to each participant by the examiners. The device was applied before sleep and removed when the patient woke up every day for 5 days. The number of EMG events per hour, the intensity of the EMG (area under EMG curve) and the total recording hours were registered.

\section{rTMS application}

Participants were tested at the same time in the afternoon (4-5 pm) each day during the 5 consecutive days of phase 2. TMS was always performed by the same examiner, who has extensive clinical experience with the TMS technique.

The participants were positioned comfortably on a physiotherapy couch in a soundproof room, and TMS was performed using a Magstim 200 stimulator (Magstim, Wales, UK) and a focal figure-8 stimulating coil (outer coil diameter $90 \mathrm{~mm}$ ). Magnetic stimuli were given at the optimum site (hot spot) for eliciting motor-evoked potentials (MEPs) with maximal amplitudes in the masseter muscle at the lowest output. The optimal site was approximately $10 \mathrm{~cm}$ lateral to the vertex and $4 \mathrm{~cm}$ anterior to the interaural line, with slight adjustments for each participant. The optimal position of the coil was then marked on the scalp with a pen to ensure consistent coil placement throughout the experiment. The coil was oriented at an angle of $45^{\circ}$ relative to the parasagittal plane with the handle pointing posteriorly. This coil orientation induces current flow in a posteroanterior direction in the underlying cortex. ${ }^{18}$

MEPs were evoked in the masseter muscle by stimulating the 'hot spot'. A light pre-contraction was obtained by asking the participants to clench their teeth slightly together during TMS. Then, the active motor threshold (AMT) in the masseter muscle was defined as the minimum stimulation intensity (at maximum stimulator output) that could induce MEPs of at least $50 \mu \mathrm{V}$ in amplitude in 5 out of 10 consecutive trials. The stimulus intensity of rTMS was set at $80 \%$ of the AMT, and the rTMS $(1 \mathrm{~Hz})$ was applied for 20 min on one side with the jaw-closing muscles at rest (see Figure 1). The same procedure was then repeated on the contralateral side.

\section{Statistics}

A two-way analysis of variance (ANOVA) with repeated measurement was used to compare EMG events per hour and EMG intensity amongst the three phases. The NRS scores of the jaw-closing muscle soreness were analysed by a non-parametric ANOVA. A post hoc Student-Newman-Keuls test was used for comparison. The mean values and standard error of the mean (SEM) are presented in the text. The level of significance was set at $5 \%$. 
a
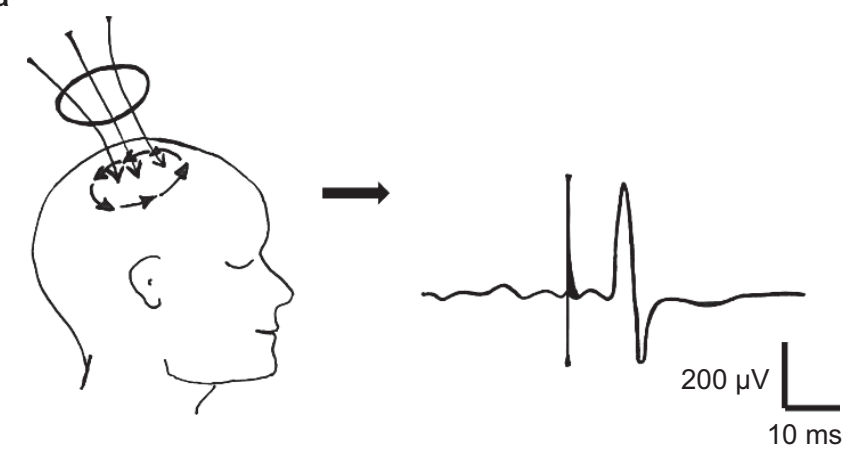

b

rTMS at $1 \mathrm{~Hz}, 80 \%$ of the AMT

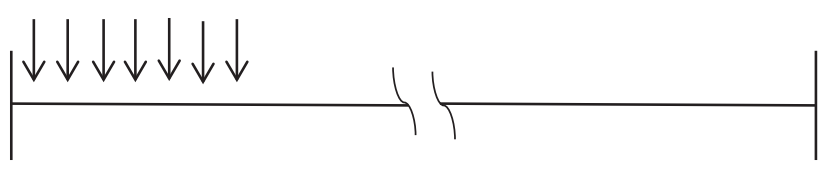

20-min bilateral

Figure 1 Repetitive transcranial magnetic stimulation rTMS application. (a) MEPs were evoked in the masseter muscle by stimulating the 'hot spot', while teeth were slightly clenched. The AMT was detected. (b) The stimulus intensity of rTMS was set at $80 \%$ of the AMT and applied at $1 \mathrm{~Hz}$ for 20 min on one side with the jaw-closing muscles at rest. The same procedure was then repeated on the contralateral side. AMT, active motor threshold; MEP, motor evoked potential; rTMS, transcranial magnetic stimulation.

\section{RESULTS}

A total of 12 patients with probable SB completed the study without any reports of side effects from rTMS. The hot spot for evoking MEPs in the masseter muscle could be identified before rTMS was applied and was located approximately $(10.2 \pm 2.1) \mathrm{cm}$ lateral to the vertex and $(4.5 \pm 1.2) \mathrm{cm}$ anterior to the interaural line. The overall AMT for the right and left masseter muscles was $68.4 \% \pm 7.8 \%$ and did not vary across the 5 consecutive days (ANOVA: $P=0.10$ ). The TMS intensity used for rTMS was set at $54.7 \% \pm 5.6 \%$.

Jaw-closing muscle activity during sleep

The EMG intensity and the number of EMG bursts per hour at baseline, during rTMS (during) and after rTMS (post) are shown in Figure $2 \mathrm{a}$ and $2 \mathrm{~b}$. The average recording hours during sleep in the three phases were $(5.9 \pm 1.3),(5.2 \pm 0.6)$ and $(5.5 \pm 0.7) \mathrm{h}$, respectively. There was no significant difference between the recording hours amongst the phases $(P>0.51)$.

The two-way ANOVA indicated that there was a significant difference in the EMG intensity amongst phases $(F=7.40, P=0.03)$. Post hoc tests showed significantly lower EMG intensities during the rTMS and follow-up phases compared to the baseline $(P=0.04, P=0.02$, respectively; Figure 2a). There were no correlations between days and sessions $(F=2.90, P=0.13)$. There were no significant differences amongst days $(F=1.44, P=0.27)$.

Regarding the number of EMG bursts, there were no significant differences amongst days $(F=0.49, P=0.74)$ or phases $(F=0.17$, $P=0.22)$ and no correlations $(F=0.90, P=0.51)$ in the number of EMG events per hour of sleep (Figure $2 b$ ).

Jaw-closing muscle soreness

The NRS scores of jaw-closing muscle soreness demonstrated significant differences amongst phases $(P<0.001)$. Post hoc tests showed a signifi-
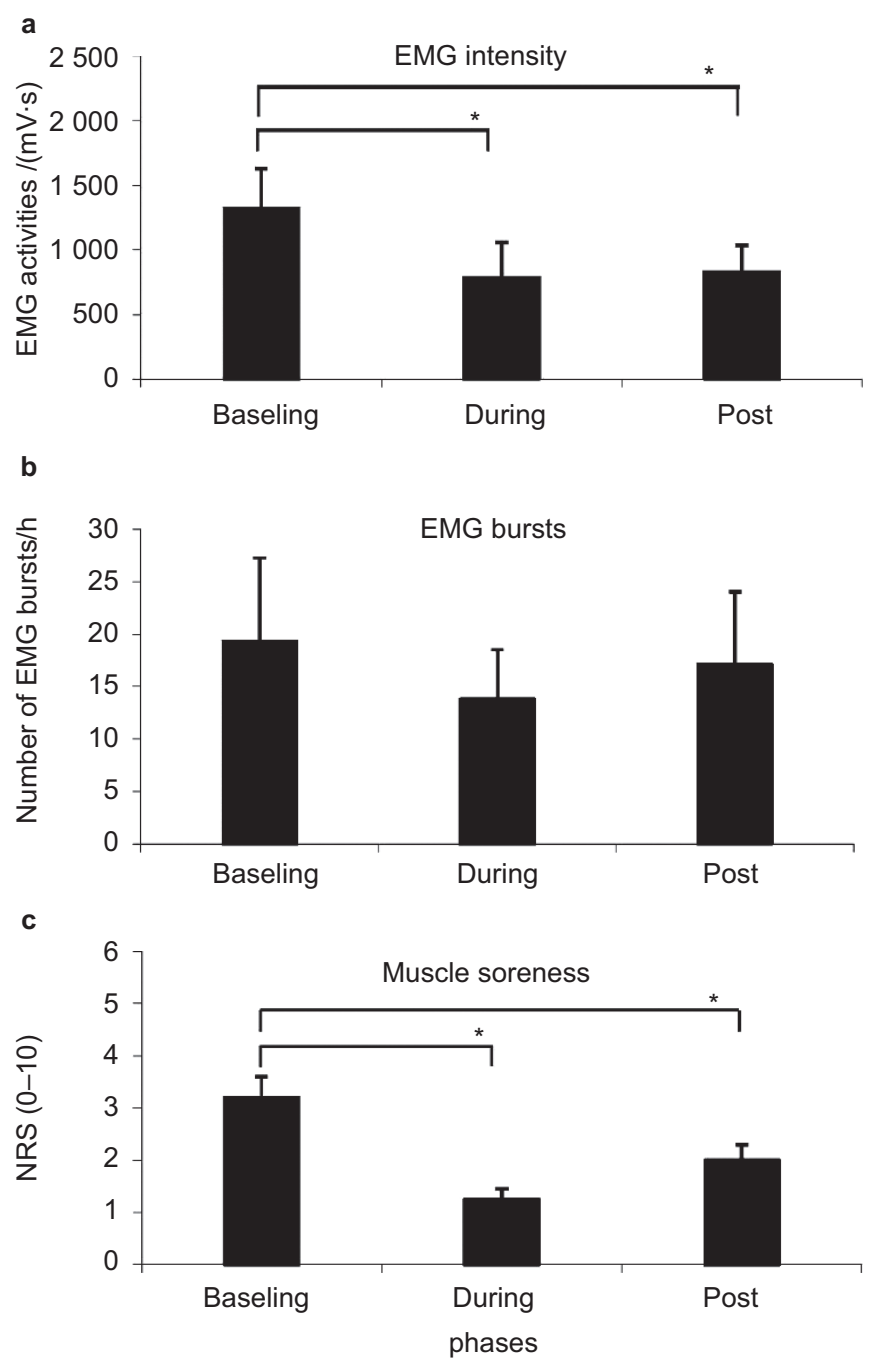

Figure 2 Effects of rTMS application. (a) Intensity of EMG activity; (b) numbers of EMG burst per hour; (c) the NRS scores of jaw-closing muscle soreness compared amongst baseline, during rTMS (during), and after rTMS (post). Mean \pm SEM. *indicates significant differences amongst the treatment phases $(P<0.05)$. EMG, electromyographic; rTMS, transcranial magnetic stimulation; SEM, standard error of arithmetic mean.

cant decrease in NRS soreness scores during rTMS and the follow-up phase compared with baseline values $(P<0.001 ; P<0.01$; Figure $2 \mathrm{c})$.

From the questionnaire data, $6 / 12(50 \%)$ participants reported less SB, 2/12 (approximately 17\%) reported no change and 4/12 (approximately 33\%) answered 'do not know' during rTMS treatment (phase 2). During the follow-up phase, 5/12 (approximately 42\%) participants still reported less SB, 3/12 (25\%) reported no change and 4/12 (approximately 33\%) reported 'do not know'.

\section{DISCUSSION}

In this pilot study with an open and non-controlled design, we applied the novel methodology of rTMS in an attempt to manage patients with probable SB. Despite the obvious limitations in the study design, we believe that the present findings, which showed a significant inhibition of the intensity of jaw-closing muscle activity during sleep along with a decrease in patients' reports of jaw-closing muscle soreness, represent an important new idea in the understanding of SB and call for further and more controlled studies. 


\section{Effects of rTMS on EMG activity during sleep}

TMS is a painless, non-invasive technique that allows investigations of the motor cortex and descending pathways in humans. ${ }^{19-20}$ In this study, we applied low-frequency rTMS $(1 \mathrm{~Hz})$ to the face motor cortex, which is the cortical representation of the masseter muscle on the primary motor cortex, to trigger transient inhibition of the descending corticobulbar projections to the trigeminal motor nuclei and brainstem reticular formations. ${ }^{21-25}$ An experimental human study has found that TMS pulses evoke descending activity in the corticobulbar axons, subsequently suppressing the excitability of the motoneuron pool. ${ }^{26}$ Interestingly, an abnormal excitability in the central masticatory pathway has been suggested in SB patients. $^{27}$

It is generally accepted that $\mathrm{SB}$ is a movement disorder with increased RMMA related to sleep arousals. ${ }^{28}$ Transient arousal is an intermediate state in which the ascending activating systems responsible for waking cortical and cardiac activity are activated but are insufficient to produce wakefulness. The genesis of RMMA is associated with a physiological sequence of micro-arousal. Patients with SB may have an increased responsiveness in their neural circuits that are responsible for the genesis of rhythmic jaw motor excitation in response to micro-arousals. ${ }^{10}$ Therefore, our data with rTMS of the cortical motor neurons suggest that the rhythmic jaw motor activation was modified to some extent and had an impact on the pathophysiology of SB. rTMS treatment may interfere with or block the neural circuits associated with $\mathrm{SB}$, therefore tending to reduce the incidence of SB events and lower the intensity of the jaw-closing muscle contractions. A notable finding from the present study was that the intensity of EMG activity was depressed significantly during rTMS treatment (Figure $2 \mathrm{a}$ ) and to a lesser but still significant degree during the follow-up phase. This suggests a reasonable short-term effect (within days) of rTMS, but longer treatment periods are needed to observe more consistent and profound long-term effects on jaw-closing muscle activation. It has been reported that rTMS-induced changes last from minutes to hours, although some reports have indicated that synaptic plasticity may last up to weeks or months after the stimulation period. ${ }^{21,29}$ Accordingly, it has been suggested that a maintenance therapy will be needed, which would consist of a priming week or weeks with daily rTMS sessions followed by maintenance sessions at longer intervals that could be used to maintain long-lasting effects. ${ }^{30-32}$ The time course of the inhibitory effects of rTMS on jaw-closing muscle activity during sleep will, however, need further studies.

\section{Effects of rTMS on jaw-closing muscle soreness}

SB may be associated with muscle symptoms such as pain or soreness. In this study, we included patients who reported no pain but had significant levels of jaw-closing muscle soreness, in particular upon awakening in the morning. rTMS has been applied to manage clinical pain conditions with variable outcomes. ${ }^{30}$ We therefore decided not to focus on pain but rather on muscle soreness in our patient population. Indeed, rTMS was associated with significant decreases in NRS scores of jaw-closing muscle soreness, an effect that persisted in the follow-up period. In line with the NRS scores, the overall perception of the treatment effects reported by the patients indicated that rTMS had significantly improved their muscle symptoms (Figure 2c). A number of explanations for this observation need to be discussed.

The decrease in NRS scores of jaw-closing muscle soreness could simply be a placebo effect, i.e., an expectation from the patients that a new, high-tech technique administered by medical experts would lead to an improvement in their symptoms. Because the study was not designed to control for placebo effects or regression towards the mean (time effects), we cannot completely rule out this possibility. Furthermore, increased cerebral blood flow and increased synaptic neurotransmission have also been shown to occur with rTMS, which may reduce stress chemicals in the brain and thus, have an indirect effect on muscle soreness and SB. ${ }^{32-33}$ Nevertheless, the observation that rTMS also leads to a significant decrease in jaw-closing muscle activity during sleep suggests that there could be a physiological effect of rTMS, although placebo mechanisms may also be mediated during sleep. ${ }^{34} \mathrm{It}$ could be speculated that rTMS could exert its effect on jaw-closing muscle soreness through the inhibition of jaw-closing muscle activity during sleep. The relation between muscle symptoms and muscle activity during sleep in SB is, however, not linear or straightforward because several reports have demonstrated that higher levels of jawclosing muscle activity during sleep are, perhaps surprisingly, associated with fewer reports of muscle symptoms. ${ }^{31,35-36}$

\section{Methodological concerns}

We recognise the limitations of an open and non-controlled study but would like to note some of the strengths of the present study. First, we used an objective measure of jaw-closing muscle activity during sleep over at least five repeated nights, rather than from a single night, to account for night-to-night variability. ${ }^{37}$ This allowed a more robust assessment of the baseline activity and the subsequent treatment effects during rTMS. It is worth noting that our statistical analyses used the data from each night but did not find significant differences across the different nights or correlations between nights and intervention. This suggests that rTMS evoked a pronounced inhibitory effect on the intensity of the jaw-closing muscle activity during sleep. The EMG-monitoring device is based on a single channel recording from the anterior temporalis muscle. Although most studies on SB have focused on the masseter muscle, there is no indication that the anterior temporalis should act differently during episodes of SB because of the high degree of functional overlap and synergistic actions.

We intended to provide more accurate data about SB than information solely based on questionnaires and clinical examinations. ${ }^{38}$ In the present study, the ambulatory EMG-recording devices were used at the subject's home for extended periods and during multiple nights. Thus, the EMG recordings did not disturb the natural quality of sleep, and the recordings could be performed for continuous nights at a relatively low cost. ${ }^{37}$

Finally, the present study only included a relatively small study sample, but the design allowed a within-subject comparison and was sufficient to detect significant differences between baseline and rTMS for the intensity of EMG activity and NRS scores of jaw-closing muscle soreness. In addition, because of the short-term follow-up design, the results do not allow speculations about EMG activity in the jawclosing muscles at a longer follow-up.

rTMS was carefully applied by only one experienced examiner. The hot spot was identified in each participant individually on each treatment day. MEP was evoked consistently before the application of rTMS to secure a specific stimulation of the relevant hot spot, i.e., the masseter muscle representation at the primary motor cortex. This study was not a PSG study, and therefore, we could only identify probable SB because no gold standard examination technique was applied. However, the strength was that we could have 5 days of continuous recordings during each of the three phases, which may not have been feasible with PSG recordings. Importantly, the present ambulatory EMG technique will be less influenced by night-to-night variations. 


\section{CONCLUSIONS}

This pilot study raises the possibility that the novel application of rTMS in patients with SB could have beneficial effects. Controlled and long-term studies with larger sample sizes are needed to replicate the findings.

\section{ACKNOWLEDGEMENTS}

This project was supported by the Priority Academic Program Development of Jiangsu Higher Education Institutions (PAPD, 2014-37) and the Medical Science and Technology Development Foundation of Jiangsu Health Department (H201338).

1 Lobbezoo F, Ahlberg J, Glaros AG et al. Bruxism defined and graded: an international consensus. J Oral Rehabil 2013; 40(1): 2-4.

2 Bader G, Lavigne G. Sleep bruxism; an overview of an oromandibular sleep movement disorder. Sleep Med Rev 2000; 4(1): 27-43.

3 Pintado MR, Anderson GC, DeLong R et al. Variation in tooth wear in young adults over a two-year period. J Prosthet Dent 1997; 77(3): 313-320.

4 Dao TT, Lund JP, Lavigne GJ. Comparison of pain and quality of life in bruxers and patients with myofascial pain of the masticatory muscles. J Orofac Pain 1994; 8(4): 350-356.

5 Lavigne GJ, Khoury S, Abe $\mathrm{S}$ et al. Bruxism physiology and pathology: an overview for clinicians. J Oral Rehabil 2008; 35(7): 476-494.

6 Lobbezoo F, Ahlberg J, Manfredini D et al. Are bruxism and the bite causally related? J Oral Rehabil 2012; 39(7): 489-501.

7 Ommerborn MA, Giraki M, Schneider $\mathrm{C}$ et al. Effects of sleep bruxism on functional and occlusal parameters: a prospective controlled investigation. Int J Oral Sci 2012; 4(3): 141-145.

8 Lavigne GJ, Huynh N, Kato T et al. Genesis of sleep bruxism: motor and autonomiccardiac interactions. Arch Oral Biol 2007; 52(4): 381-384.

9 Kervancioglu BB, Teismann IK, Rain M et al. Sensorimotor cortical activation in patients with sleep bruxism. J Sleep Res 2012; 21(5): 507-514.

10 Kato T, Montplaisir JY, Guitard F et al. Evidence that experimentally induced sleep bruxism is a consequence of transient arousal. J Dent Res 2003; 82(4): 284-288.

11 Lobbezoo F, van der Zaag J, van Selms MK et al. Principles for the management of bruxism. J Oral Rehabil 2008; 35(7): 509-523.

12 Huynh N, Manzini C, Rompré PH et al. Weighing the potential effectiveness of various treatments for sleep bruxism. J Can Dent Assoc 2007; 73(8): 727-730.

13 Rothwell JC. Techniques and mechanisms of action of transcranial stimulation of the human motor cortex. J Neurosci Methods 1997; 74(2): 113-122.

14 Aleman A. Use of repetitive transcranial magnetic stimulation for treatment in psychiatry. Clin Psychopharmacol Neurosci 2013; 11(2): 53-59.

15 Theodoroff SM, Folmer RL. Repetitive transcranial magnetic stimulation as a treatment for chronic tinnitus: a critical review. Otol Neurotol2013; 34(2): 199-208.

16 Conti PC, Stuginski-Barbosa J, Bonjardim LR et al. Contingent electrical stimulation inhibits jaw muscle activity during sleep but not pain intensity or masticatory muscle pressure pain threshold in self-reported bruxers: a pilot study. Oral Surg Oral Med Oral Pathol Oral Radiol 2014; 117(1): 45-52.

17 Jadidi F, Castrillon E, Svensson P. Effect of conditioning electrical stimuli on temporalis electromyographic activity during sleep. J Oral Rehabil 2008; 35(3): 171-183.

18 Jaberzadeh S, Sakuma S, Zoghi M et al. Focal transcranial magnetic stimulation of motor cortex evokes bilateral and symmetrical silent periods in human masseter muscles. Clin Neurophysiol 2008; 119(3): 693-703.
19 Hallett M. Transcranial magnetic stimulation: a primer. Neuron 2007; 55(2): 187 199.

20 Ortu E, Deriu F, Suppa A et al. Intracortical modulation of cortical-bulbar responses for the masseter muscle. J Physiol 2008; 586(14): 3385-3404.

21 Nordstrom MA. Insights into the bilateral cortical control of human masticatory muscles revealed by transcranial magnetic stimulation. Arch Oral Biol 2007; 52(4): 338-342.

22 Sowman PF, Flavel SC, McShane CL et al. Transcranial magnetic stimulation reduces masseter motoneuron pool excitability throughout the cortical silent period. Clin Neurophysiol 2008; 119(5): 1119-1129.

23 Siebner HR, Filipovic SR, Rowe JB et al. Patients with focal arm dystonia have increased sensitivity to slow-frequency repetitive TMS of the dorsal premotor cortex. Brain 2003; 126(Pt 12): 2710-2725.

24 Hoffman RE, Cavus I. Slow transcranial magnetic stimulation, long-term depotentiation, and brain hyperexcitability disorders. Am J Psychiatry 2002; 159(7): 10931102 .

25 Lee M, Kim SE, Kim WS et al. Cortico-cortical modulation induced by 1 - Hz repetitive transcranial magnetic stimulation of the temporal cortex. J Clin Neurol 2013; 9(2): 75-82.

26 Zhang $Y$, Boudreau $S$, Wang $M$ et al. Effects of periodontal afferent inputs on corticomotor excitability in humans. J Oral Rehabil 2010; 37(1): 39-47.

27 Huang $\mathrm{H}$, Song $\mathrm{YH}$, Wang JJ et al. Excitability of the central masticatory pathways in patients with sleep bruxism. Neurosci Lett 2014; 558: 82-86.

28 Halász P, Terzano M, Parrino L et al. The nature of arousal in sleep. J Sleep Res 2004, 13(1): 1-23.

29 Pell GS, Roth Y, Zangen A. Modulation of cortical excitability induced by repetitive transcranial magnetic stimulation: influence of timing and geometrical parameters and underlying mechanisms. Prog Neurobiol 2011; 93(1): 59-98.

30 Treister R, Lang M, Klein MM et al. Non-invasive transcranial magnetic stimulation (TMS) of the motor cortex for neuropathic pain-at the tipping point? Rambam Maimonides Med J 2013; 4(4): e0023.

31 Rompré PH, Daigle-Landry D, Guitard F et al. Identification of a sleep bruxism subgroup with a higher risk of pain. J Dent Res 2007; 86(9): 837-842.

32 Mesquita RC, Faseyitan OK, Turkeltaub PE et al. Blood flow and oxygenation changes due to low-frequency repetitive transcranial magnetic stimulation of the cerebral cortex. J Biomed Opt 2013; 18(6): 067006

33 Funke K, Benali A. Modulation of cortical inhibition by rTMS --findings obtained from animal models. J Physiol 2011; 589(Pt 18): 4423-4435.

34 Lavigne GJ, Soucy JP, Lobbezoo F et al. Double-blind, crossover, placebo-controlled trial of bromocriptine in patients with sleep bruxism. Clin Neuropharmacol 2001; 24(3): 145-149.

35 Lavigne GJ, Rompré PH, Montplaisir JY et al. Motor activity in sleep bruxism with concomitant jaw muscle pain. A retrospective pilot study. Eur J Oral Sci 1997; 105(1): 92-95.

36 Arima T, Svensson P, Rasmussen C et al. The relationship between selective sleep deprivation, nocturnal jaw-muscle activity and pain in healthy men. J Oral Rehabil 2001; 28(2): 140-148.

37 Jadidi F, Nørregaard O, Baad-Hansen L et al. Assessment of sleep parameters during contingent electrical stimulation in subjects with jaw muscle activity during sleep: a polysomnographic study. Eur J Oral Sci 2011; 119(3): 211-218.

38 Svensson P, Baad-Hansen L, Pigg M et al. Guidelines and recommendations for assessment of somatosensory function in oro-facial pain conditions - a taskforce report. J Oral Rehabil 2011; 38(5): 366-394.

\footnotetext{
(c) (i) (2) (2) This license allows readers to copy, distribute and transmit the Contribution as cc) transform or build upon the Contribution as long as the resulting work is then distributed under this is a similar license. Readers are not permitted to use the Contribution for commercial purposes. Please read the full license for further details at - http://creativecommons.org/ licenses/by-nc-sa/4.0/
} 